\title{
Interventions for preventing falls in Parkinson's disease (Protocol)
}

Canning CG, Allen NE, Bloem BR, Keus SHJ, Munneke M, Nieuwboer A, Sherrington C, Verheyden GSAF

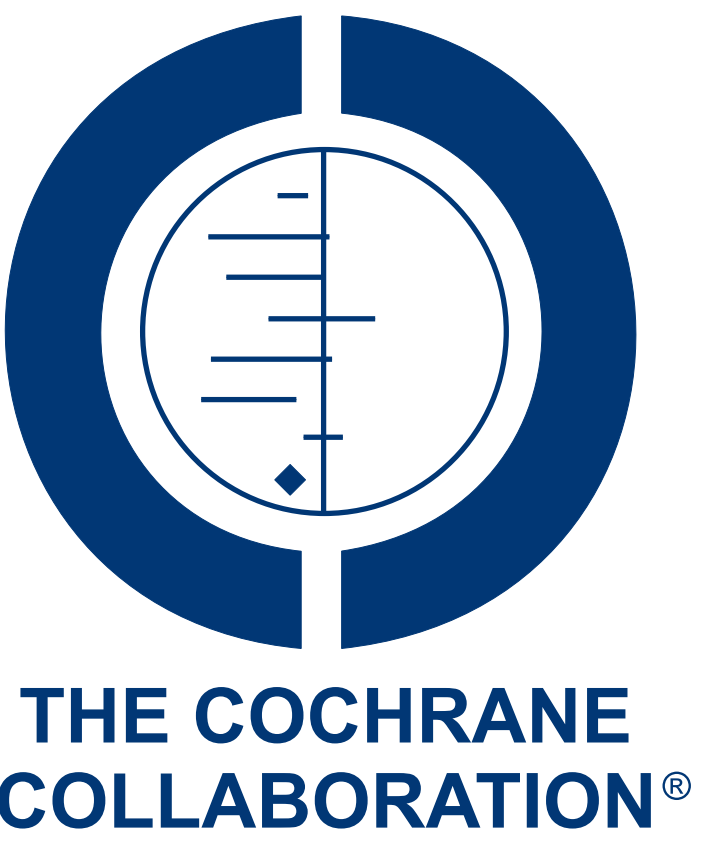

This is a reprint of a Cochrane protocol, prepared and maintained by The Cochrane Collaboration and published in The Cochrane Library 2015, Issue 3

http://www.thecochranelibrary.com

\section{WILEY}

Interventions for preventing falls in Parkinson's disease (Protocol)

Copyright @ 2015 The Cochrane Collaboration. Published by John Wiley \& Sons, Ltd. 
TABLE OF CONTENTS

HEADER . . . . . . . . . . . . . . . . . . . . . . . . . . . . . . . . . . . . 1

ABSTRACT . . . . . . . . . . . . . . . . . . . . . . . . . . . . . . . . . . . . . . . . . . . . .

BACKGROUND . . . . . . . . . . . . . . . . . . . . . . . . . . . . . . . . . . . . .

OBJECTIVES . . . . . . . . . . . . . . . . . . . . . . . . . . . . . . . . . . . . . . .

METHODS . . . . . . . . . . . . . . . . . . . . . . . . . . . . . . . . . . . . . .

REFERENCES . . . . . . . . . . . . . . . . . . . . . . . . . . . . . . . . . . . . . . 5

APPENDICES . . . . . . . . . . . . . . . . . . . . . . . . . . . . . . . . . . . . . . 6

CONTRIBUTIONS OF AUTHORS . . . . . . . . . . . . . . . . . . . . . . . . . . . . . . . . . . . . . . . . . . . . .

DECLARATIONS OF INTEREST . . . . . . . . . . . . . . . . . . . . . . . . . . . . . . . . . . . . . 7 


\title{
[Intervention Protocol]
}

\section{Interventions for preventing falls in Parkinson's disease}

\author{
Colleen G Canning ${ }^{1}$, Natalie E Allen ${ }^{1}$, Bastiaan R Bloem ${ }^{2}$, Samyra HJ Keus ${ }^{2}$, Marten Munneke ${ }^{2}$, Alice Nieuwboer ${ }^{3}$, Catherine \\ Sherrington ${ }^{4}$, Geert SAF Verheyden ${ }^{3}$ \\ ${ }^{1}$ Clinical and Rehabilitation Sciences Research Group - Faculty of Health Sciences, University of Sydney, Lidcombe, Australia. \\ ${ }^{2}$ Department of Neurology, Radboud University Nijmegen Medical Center, Nijmegen, Netherlands. ${ }^{3}$ Department of Rehabilitation \\ Sciences, KU Leuven, Leuven, Belgium. ${ }^{4}$ Musculoskeletal Division, The George Institute for Global Health, Sydney Medical School, \\ University of Sydney, Sydney, Australia
}

Contact address: Colleen G Canning, Clinical and Rehabilitation Sciences Research Group - Faculty of Health Sciences, University of Sydney, PO Box 170, Lidcombe, NSW, 1825, Australia. colleen.canning@sydney.edu.au.

Editorial group: Cochrane Movement Disorders Group.

Publication status and date: Edited (no change to conclusions), published in Issue 3, 2015.

Citation: Canning CG, Allen NE, Bloem BR, Keus SHJ, Munneke M, Nieuwboer A, Sherrington C, Verheyden GSAF. Interventions for preventing falls in Parkinson's disease. Cochrane Database of Systematic Reviews 2015, Issue 3. Art. No.: CD011574. DOI: 10.1002/14651858.CD011574.

Copyright (C) 2015 The Cochrane Collaboration. Published by John Wiley \& Sons, Ltd.

\section{A B S T R A C T}

This is the protocol for a review and there is no abstract. The objectives are as follows:

To assess the effects of interventions designed to reduce the incidence of falls in people with Parkinson's disease.

\section{B A C K G R O U N D}

\section{Description of the condition}

People with Parkinson's disease (PD) fall frequently and recurrently with approximately $60 \%$ of individuals falling each year and two thirds of these people falling recurrently (Allen 2013; Bloem 2001; Latt 2009; Paul 2013; Pickering 2007). These rates are double those reported for the general older population (Gillespie 2012). In addition, falls in people with PD are associated with injury (Walker 2013; Wielinski 2005) with the incidence of hip fracture reported to be four times that of older people of the same age without PD. Not surprising, falls are associated with escalating healthcare costs (Pressley 2003) and are major contributors to reduced health-related quality of life (Rahman 2008; Soh 2011). A large number of fall risk factors have been identified in people with PD. Consistently identified risk factors include a his- tory of past falls (Allcock 2009; Latt 2009; Paul 2013; Pickering 2007); disease severity (Allcock 2009; Kerr 2010; Latt 2009; Paul 2013; Pickering 2007); freezing of gait (Kerr 2010; Latt 2009; Paul 2013); balance, mobility and lower limb muscle strength deficits (Kerr 2010; Latt 2009; Paul 2013); and cognitive deficits (Allcock 2009; Latt 2009; Paul 2013). Whilst falls history and disease severity are fixed, the remaining risk factors have the potential to be modified with exercise or movement strategy training (Allen 2011; Tomlinson 2013), which may in turn reduce falls. While falls are commonly monitored as adverse events in intervention trials (Deuschl 2007; Nieuwboer 2007; van Nimwegen 2013), only recently have interventions designed primarily to reduce falls in people with PD been developed and investigated (Ashburn 2007; Canning 2009; Chung 2010; Fletcher 2012; Goodwin 2011; Li 2012; Mirelman 2011; Morris 2011).

Interventions for preventing falls in Parkinson's disease (Protocol)

Copyright $\odot 2015$ The Cochrane Collaboration. Published by John Wiley \& Sons, Ltd. 


\section{Description of the intervention}

Interventions designed to reduce falls in people with PD include exercise and/or movement strategy training, pharmacological and/ or surgical management, environmental modifications and any other intervention designed to reduce falls in this population. Interventions are classified as single interventions (e.g., exercise), multiple interventions (e.g., exercise plus environmental modifications) or multifactorial interventions (i.e., multiple interventions tailored to the individual's identified risk factors).

\section{Why it is important to do this review}

Recently, a number of large-scale randomised controlled trials and several smaller trials specifically testing interventions designed to reduce falls in people with PD have been published. In addition, participants with PD are no longer included in the recent update of the Cochrane review of interventions for preventing falls in older people living in the community (Gillespie 2012). Further, while falls as an outcome is addressed in recent updates of Cochrane reviews of physiotherapy interventions for PD (Tomlinson 2013 ; Tomlinson 2014 ), these reviews do not differentiate between physiotherapy interventions primarily designed to reduce falls versus interventions primarily designed to improve mobility. Also, the scope of the physiotherapy reviews is limited to physical interventions. Therefore, there is a need to systematically review the literature to identify trials of all interventions aimed at reducing falls in people with PD and summarise this evidence for people with PD, clinicians, researchers and policy makers.

\section{O B JECT IVES}

To assess the effects of interventions designed to reduce the incidence of falls in people with Parkinson's disease.

\section{METHODS}

\section{Criteria for considering studies for this review}

\section{Types of studies}

We will include randomised controlled trials and quasi-randomised trials, including randomised and quasi-randomised cluster and cross-over trials. Should any cross-over trial meet our inclusion criteria, we will include data from the first phase only to minimise the risk of carry-over effects of the interventions. We will not include studies published only in abstract form.

\section{Types of participants}

We will include trials of participants with idiopathic Parkinson's disease (PD) who have been diagnosed by the UK Parkinson's Disease Brain Bank criteria (Hughes 1992) or by a clinical definition. No restrictions will be made with regard to gender, age or disease duration. We will include trials reporting an intervention carried out in a mixed sample of participants, including people with idiopathic $\mathrm{PD}$, if separate data are available for participants with PD.

\section{Types of interventions}

We will include any intervention where a stated primary or secondary aim is to reduce falls in people with PD. Therefore, any intervention which is not aimed at preventing falls, and which reports falls as an adverse event, will not be included. We will include trials where a fall-prevention intervention is compared with 'usual care', a 'placebo' control intervention, or another fall-prevention intervention.

\section{Types of outcome measures}

We will only include trials that report rate or number of falls, or number of participants sustaining at least one fall during the follow-up. The preferred method of reporting is prospective daily calendars returned monthly from randomisation (Lamb 2005). However, we will also include trials that record falls retrospectively.

\section{Primary outcomes}

- Rate (number) of falls

- Number of fallers

\section{Secondary outcomes}

- Number of participants sustaining fall-related fractures

- Number and type of adverse events

- Quality of life

- Economic outcomes

\section{Search methods for identification of studies}

\section{Electronic searches}

We will search the Cochrane Movement Disorders Group Trials Register, the Cochrane Central Register of Controlled Trials (CENTRAL) (The Cochrane Library, latest issue), MEDLINE (1946 to present), EMBASE (1947 to present), CINAHL (Cumulative Index to Nursing and Allied Health Literature) (1982 to present), PsycINFO (1806 to present), AMED (1985 to present), the Physiotherapy Evidence Database (PEDro) and online trial registers. 
The full search strategy can be found in Appendix 1 .

\section{Searching other resources}

To identify any further published or ongoing trials, we will:

1. search trial registers: ClinicalTrials.gov (http://

clinicaltrials.gov/), Current Controlled Trials (www.controlledtrials.com/), and International Clinical Trials Registry Platform Search Portal (http://apps.who.int/trialsearch/)

2. check reference lists of relevant articles

3. contact trialists and researchers in the field

4. use Science Citation Index Cited Reference Search

5. check studies included in the Cochrane review of interventions for preventing falls in older people living in the community (Gillespie 2012) and the Cochrane review of interventions for preventing falls in older people in care facilities and hospitals (Cameron 2012) for any trial which includes a subgroup of people with PD.

We will not apply any language restrictions.

\section{Data collection and analysis}

\section{Selection of studies}

$\mathrm{CC}$ and NA will separately screen the search results (title, abstract and descriptors) to identify studies for possible inclusion. We will resolve disagreements through discussion. After initial screening, CC and NA will separately assess the eligibility of trials based on full text. Again, we will resolve disagreements through discussion. We will contact study authors for additional information if necessary.

\section{Data extraction and management}

Two pairs of review authors will independently extract data using a pre-tested data extraction form. Disagreement will be resolved by consensus or third-party adjudication.

\section{Assessment of risk of bias in included studies}

CC and SK will independently assess risk of bias using the recommendations in the Cochrane Handbook for Systematic Reviews of Interventions (Higgins 2011a). Review authors will not be blinded to author and source institution and they will not assess their own trials. Disagreement will be resolved by consensus or third party adjudication.

We will assess the following domains: random sequence generation (selection bias); allocation concealment (selection bias); blinding of participants and personnel (performance bias); blinding of outcome assessment (detection bias) for falls and fallers, and for fractures separately; incomplete outcome data (attrition bias) for falls and fallers separately. We will also assess bias in the recall of falls due to unreliable methods of ascertainment (Hannan 2010). We will use the criteria developed by Gillespie 2012 for judging risk of bias in fall prevention trials. We will also use the specific criteria for assessing attrition bias in falls trials developed by Gillespie 2012 . We will collect this information on the data extraction sheet and resolve disagreements through discussion.

\section{Measures of treatment effect}

We will report treatment effect for rate of falls as a rate ratio (RaR) and $95 \%$ confidence interval (CI), where rate of falls is the total number of falls per unit of person time that falls were monitored (e.g. falls per person year). We will use the RaR and 95\% CI if these are reported. If both adjusted and unadjusted RaRs are reported, we will use the unadjusted $\mathrm{RaR}$, unless the adjustment was for clustering. If a $\mathrm{RaR}$ is not reported but appropriate raw data are available, we will calculate a RaR and 95\% CI (see Gillespie 2012). It is possible that individual multiple fallers may have excessive influence on the rate of falls results. To investigate this possibility, we will record procedures used by investigators to decrease this risk, such as randomisation stratified by fall history or analyses adjusted for previous falls. We will also extract baseline falling rates for each group (where available). This information will be presented in a table.

For number of fallers and number of participants sustaining fallrelated fractures, we will report a risk ratio (RR) and 95\% CI. If trials monitor falls for longer than one year, we will use results reported at one year if these are available.

For the quality of life, we will calculate mean differences (MD) or standardised mean differences (SMD) for continuous outcomes.

\section{Unit of analysis issues}

We will incorporate data from cluster-randomised trials and will perform adjustments for clustering (Higgins 2011b) if this has not been presented in the published report. If no estimate of the intraclass correlation coefficient (ICC) is available, we will use an ICC of 0.01 as reported by Smeeth 2002 .

We will incorporate trials with more than one intervention arm compared with a control group. To avoid 'double counting' of control participants from these trials, the total falls and participant numbers in the control group will be allocated in proportion to the participant numbers in each intervention arm.

\section{Dealing with missing data}

We will provide an overview of missing data from our selected studies in a table format. We will not use a cut-off as an inclusion criterion. We will contact the study authors to acquire data. We will address the potential impact of missing data in the assessment of risk of bias in the discussion of the review. 


\section{Assessment of heterogeneity}

We will assess heterogeneity by visual inspection of forest plots, as well as considering both the $\mathrm{Chi}^{2}$ test (with statistical significance set at $\mathrm{P}<0.10$ ) and the $\mathrm{I}^{2}$ statistic. Depending on the degree of heterogeneity found, we may decide against reporting pooled results and present forest plots only without pooled statistics together with a narrative description of the results of our review. We will carry out analyses of subgroups of studies to determine whether heterogeneity is explained by study and/or participant characteristics.

\section{Assessment of reporting biases}

We will minimise reporting bias by comprehensively searching multiple databases, searching for studies in languages other than English, and searching the grey literature and trial registries. We will observe funnel plots and consider reporting bias as a possible explanation for findings in the discussion of the review.

\section{Data synthesis}

We will perform separate analyses for trials comparing an active fall-prevention intervention with 'usual care', or with a 'placebo' control intervention, and trials comparing two active fall-prevention interventions.

We will group interventions using the fall prevention classification taxonomy developed by the Prevention of Falls Network Europe (ProFaNE) (Lamb 2007). Interventions are classified according to intervention type: exercises, medication (drug target, i.e., withdrawal, dose reduction or increase, substitution, provision), surgery, management of urinary incontinence, fluid or nutrition therapy, psychological interventions, environment/assistive technology, social environment, interventions to increase knowledge, other interventions. Interventions are also classified according to combination of intervention types: single, multiple (more than one intervention type) or multifactorial (more than one intervention type specifically targeting person-specific identified fall risk factors). Full details are available in the ProFaNE Taxonomy Manual (Lamb 2007).

Within these categories, we will group the results of trials with comparable interventions and participant characteristics and compile forest plots using the generic inverse variance method in Review Manager software (RevMan 5.3). We will calculate pooled RaRs for falls and pooled RRs for fallers, fracture, and adverse events with 95\% CIs using the fixed-effect model. Where there is substantial clinical or statistical heterogeneity we will pool the data using the random-effects model.

If we cannot carry out meta-analysis because of substantial differences between studies or when there is only one study identified, we will present results in a forest plot and provide a narrative review.

\section{Subgroup analysis and investigation of heterogeneity}

If possible, we will carry out subgroup analyses to determine whether outcomes vary according to disease severity or level of fall risk. For exercise trials, subgroup analysis will be undertaken for type of exercise and amount of supervised exercise.

We will use the random-effects model to pool data in all analyses testing for subgroup differences due to the high risk of falsepositive results when comparing subgroups in a fixed-effect model (Higgins 2011c). We will use the test for subgroup differences available in RevMan 5.3 to determine whether there is evidence of a difference in treatment effect between subgroups.

\section{Economic evaluation}

Where comprehensive economic evaluations are incorporated in the included studies, we will report the incremental cost per fall prevented and per quality of life year (QALY) gained by the intervention compared with the comparator group, as stated by the authors. We will also extract from trials reporting a cost analysis or cost description, the type of resource use (e.g. delivering the intervention, hospital admissions, outpatient visits) and the cost of the item for each group.

\section{Sensitivity analysis}

We will perform sensitivity analyses to explore the impact of risk of bias on pooled estimates of treatment effect. We will remove trials from pooled analyses if they are assessed as having high risk of bias in one or more key domains: random sequence generation (selection bias), allocation concealment (selection bias), blinding of outcome assessors (detection bias), and incomplete outcome data (attrition bias) (see Higgins 2011a: Table 8.7.a). We will also consider performing sensitivity analyses to explore the impact of fall monitoring time. We will remove trials from pooled analyses if they monitor falls for less than three months. 


\section{R E F E R E N C E S}

\section{Additional references}

\section{Allcock 2009}

Allcock LM, Rowan EN, Steen IN, Wesnes K, Kenny RA, Burn DJ. Impaired attention predicts falling in Parkinson's disease. Parkinsonism \& Related Disorders 2009;15(2): 110-5. [PUBMED: 18487069]

Allen 2011

Allen NE, Sherrington C, Paul SS, Canning CG. Balance and falls in Parkinson's disease: a meta-analysis of the effect of exercise and motor training. Movement Disorders 2011; 26(9):1605-15. [PUBMED: 21674624]

\section{Allen 2013}

Allen NE, Schwarzel AK, Canning CG. Recurrent falls in Parkinson's disease: a systematic review. Parkinson's Disease 2013:906274. [PUBMED: 23533953]

Ashburn 2007

Ashburn A, Fazakarley L, Ballinger C, Pickering R, McLellan LD, Fitton C. A randomised controlled trial of a home based exercise programme to reduce the risk of falling among people with Parkinson's disease. Journal of Neurology, Neurosurgery, and Psychiatry 2007;78(7):678-84. [PUBMED: 17119004]

Bloem 2001

Bloem BR, Grimbergen YA, Cramer M, Willemsen $\mathrm{M}$, Zwinderman AH. Prospective assessment of falls in Parkinson's disease. Journal of Neurology 2001;248(11): 950-8. [PUBMED: 11757958]

\section{Cameron 2012}

Cameron ID, Gillespie LD, Robertson MC, Murray GR, Hill KD, Cumming RG, et al. Interventions for preventing falls in older people in care facilities and hospitals. Cochrane Database of Systematic Reviews 2012, Issue 12. [DOI: 10.1002/14651858.CD005465.pub3]

\section{Canning 2009}

Canning CG, Sherrington C, Lord SR, Fung VS, Close JC, Latt MD, et al. Exercise therapy for prevention of falls in people with Parkinson's disease: a protocol for a randomised controlled trial and economic evaluation. BMC Neurology 2009;9:4. [PUBMED: 19161631]

\section{Chung 2010}

Chung KA, Lobb BM, Nutt JG, Horak FB. Effects of a central cholinesterase inhibitor on reducing falls in Parkinson disease. Neurology 2010;75(14):1263-9. [PUBMED: 20810998]

\section{Deuschl 2007}

Deuschl G, Vaitkus A, Fox GC, Roscher T, Schremmer D, Gordin A. Efficacy and tolerability of Entacapone versus Cabergoline in parkinsonian patients suffering from wearing-off. Movement Disorders 2007;22(11):1550-5. [PUBMED: 17516484]

\section{Fletcher 2012}

Fletcher E, Goodwin VA, Richards SH, Campbell JL, Taylor RS. An exercise intervention to prevent falls in Parkinson's: an economic evaluation. BMC Health Services Research 2012;12:426. [PUBMED: 23176532]

\section{Gillespie 2012}

Gillespie LD, Robertson MC, Gillespie WJ, Sherrington C, Gates $S$, Clemson LM, et al. Interventions for preventing falls in older people living in the community. Cochrane Database of Systematic Reviews 2012, Issue 9. [DOI: 10.1002/14651858.CD007146.pub3]

\section{Goodwin 2011}

Goodwin VA, Richards SH, Henley W, Ewings P, Taylor AH, Campbell JL. An exercise intervention to prevent falls in people with Parkinson's disease: a pragmatic randomised controlled trial. Journal of Neurology, Neurosurgery, and Psychiatry 2011;82(11):1232-8. [PUBMED: 21856692]

\section{Hannan 2010}

Hannan MT, Gagnon MM, Aneja J, Jones RN, Cupples LA, Lipsitz LA, et al. Optimizing the tracking of falls in studies of older participants: comparison of quarterly telephone recall with monthly falls calendars in the MOBILIZE Boston Study. American Journal of Epidemiology 2010;171 (9):1031-6. [PUBMED: 20360242]

\section{Higgins 2011a}

Higgins JPT, Altman DG, Sterne JAC (editors). Chapter 8: Assessing risk of bias in included studies. In: Higgins JPT, Green S editor(s). Cochrane Handbook forSystematic Reviews of Interventions Version 5.1.0 (updated March 2011). The Cochrane Collaboration, 2011. Available from www.cochrane-handbook.org.

Higgins 2011b

Higgins JPT, Deeks JJ, Altman DG. Chapter 16.3.4: Approximate analyses of cluster-randomized trials for metaanalysis: effective sample sizes. Cochrane Handbook for Systematic Reviews of Interventions Version 5.1.0 (updated March 2011). The Cochrane Collaboration, 2011. Available from www.cochrane-handbook.org.

\section{Higgins 2011c}

Deeks JJ, Higgins JPT, Altman DG. Chapter 9.6.3.1: Is the effect different in different subgroups?. In: Higgins JPT, Green S editor(s). Cochrane Handbook for Systematic Reviews of Interventions. The Cochrane Collaboration, 2011. Available from www.cochrane-handbook.org.

\section{Hughes 1992}

Hughes AJ, Daniel SE, Kilford L, Lees AJ. Accuracy of clinical diagnosis of idiopathic Parkinson's disease: a clinico-pathological study of 100 cases. Journal of Neurology, Neurosurgery, and Psychiatry 1992;55(3):181-4. [PUBMED: 1564476]

Kerr 2010

Kerr GK, Worringham CJ, Cole MH, Lacherez PF, Wood JM, Silburn PA. Predictors of future falls in Parkinson disease. Neurology 2010;75(2):116-24. [PUBMED: 20574039] 


\section{Lamb 2005}

Lamb SE, Jorstad-Stein EC, Hauer K, Becker C. Development of a common outcome data set for fall injury prevention trials: the Prevention of Falls Network Europe consensus. Journal of the American Geriatrics Society 2005; 53(9):1618-22. [PUBMED: 16137297]

\section{Lamb 2007}

Lamb SE, Hauer K, Becker C. ProFaNE Taxonomy : Manual for the fall prevention classification system. www.profane.eu.org/documents/Falls_Taxonomy.pdf (accessed 24 October 2013) 2007.

\section{Latt 2009}

Latt MD, Lord SR, Morris JG, Fung VS. Clinical and physiological assessments for elucidating falls risk in Parkinson's disease. Movement Disorders 2009;24(9): 1280-9. [PUBMED: 19425059]

\section{Li 2012}

Li F, Harmer P, Fitzgerald K, Eckstrom E, Stock R, Galver $\mathrm{J}$, et al. Tai chi and postural stability in patients with Parkinson's disease. New England Journal of Medicine 2012; 366(6):511-9. [PUBMED: 22316445]

\section{Mirelman 2011}

Mirelman A, Maidan I, Herman T, Deutsch JE, Giladi N, Hausdorff JM. Virtual reality for gait training: can it induce motor learning to enhance complex walking and reduce fall risk in patients with Parkinson's disease?. Journals of Gerontology. Series A, Biological Sciences and Medical Sciences 2011;66(2):234-40. [PUBMED: 21106702]

\section{Morris 2011}

Morris ME, Menz HB, McGinley JL, Huxham FE, Murphy AT, Iansek R, et al. Falls and mobility in Parkinson's disease: protocol for a randomised controlled clinical trial. $B M C$ Neurology 2011;11:93. [PUBMED: 21801451]

Nieuwboer 2007

Nieuwboer A, Kwakkel G, Rochester L, Jones D, van Wegen E, Willems AM, et al. Cueing training in the home improves gait-related mobility in Parkinson's disease: the RESCUE trial. Journal of Neurology, Neurosurgery, and Psychiatry 2007;78(2):134-40. [PUBMED: 17229744]

Paul 2013

Paul SS, Canning CG, Sherrington C, Lord SR, Close JC, Fung VS. Three simple clinical tests to accurately predict falls in people with Parkinson's disease. Movement Disorders 2013;28(5):655-62. [PUBMED: 23450694]

\section{Pickering 2007}

Pickering RM, Grimbergen YA, Rigney U, Ashburn A, Mazibrada G, Wood B, et al. A meta-analysis of six prospective studies of falling in Parkinson's disease. Movement Disorders 2007;22(13):1892-900. [PUBMED: 17588236]

\section{Pressley 2003}

Pressley JC, Louis ED, Tang MX, Cote L, Cohen PD, Glied $S$, et al. The impact of comorbid disease and injuries on resource use and expenditures in parkinsonism. Neurology 2003;60(1):87-93. [PUBMED: 12525724]

Rahman 2008

Rahman S, Griffin HJ, Quinn NP, Jahanshahi M. Quality of life in Parkinson's disease: the relative importance of the symptoms. Movement Disorders 2008;23(10):1428-34. [PUBMED: 18543333]

RevMan 5.3

The Nordic Cochrane Centre, The Cochrane Collaboration. Review Manager (RevMan). 5.3. Copenhagen: The Nordic Cochrane Centre, The Cochrane Collaboration, 2012.

\section{Smeeth 2002}

Smeeth L, Ng ES. Intraclass correlation coefficients for cluster randomized trials in primary care: data from the MRC Trial of the Assessment and Management of Older People in the Community. Controlled Clinical Trials 2002; 23(4):409-21. [PUBMED: 12161083]

\section{Soh 2011}

Soh SE, Morris ME, McGinley JL. Determinants of healthrelated quality of life in Parkinson's disease: a systematic review. Parkinsonism \& Related Disorders 2011;17(1):1-9. [PUBMED: 20833572]

\section{Tomlinson 2013}

Tomlinson CL, Patel S, Meek C, Herd CP, Clarke CE, Stowe $\mathrm{R}$, et al. Physiotherapy versus placebo or no intervention in Parkinson's disease. The Cochrane database of systematic reviews 2013;9:CD002817. [PUBMED: 24018704]

\section{Tomlinson 2014}

Tomlinson CL, Herd CP, Clarke CE, Meek C, Patel S, Stowe R, et al. Physiotherapy for Parkinson's disease: a comparison of techniques. Cochrane Database of Systematic Reviews 2014, Issue 6. [DOI: 10.1002/ 14651858.CD002815.pub2]

\section{van Nimwegen 2013}

van Nimwegen M, Speelman AD, Overeem $S$, van de Warrenburg BP, Smulders K, Dontje ML, et al. Promotion of physical activity and fitness in sedentary patients with Parkinson's disease: randomised controlled trial. $B M J$ (Clinical research ed.) 2013;346:f576. [PUBMED: 23457213]

\section{Walker 2013}

Walker RW, Chaplin A, Hancock RL, Rutherford R, Gray WK. Hip fractures in people with idiopathic Parkinson's disease: incidence and outcomes. Movement Disorders 2013; 28(3):334-40. [PUBMED: 23389925]

\section{Wielinski 2005}

Wielinski CL, Erickson-Davis C, Wichmann R, WaldeDouglas M, Parashos SA. Falls and injuries resulting from falls among patients with Parkinson's disease and other parkinsonian syndromes. Movement Disorders 2005;20(4): 410-5. [PUBMED: 15580552]

* Indicates the major publication for the study 


\section{A P P E N D I C E S}

\section{Appendix I. MEDLINE search strategy}

We will use the following strategy for MEDLINE (Ovid) and adapt it to search the other databases:

1. Accidental Falls/

2. (falls or faller $\$ 1$ ).tw.

3. 1 or 2

4. Parkinson Disease/

5. Parkinson*.ti.

6. Parkinson*.ab.

7. PD.ti.

8. PD.ab.

9. 4 or 5 or 6 or 7 or 8

10.3 and 9

11. randomized controlled trial.pt.

12. randomized.ab.

13. placebo.ab.

14. drug therapy.fs.

15. randomly.ab.

16. trial.ab.

17. groups.ab.

18. 11 or 12 or 13 or 14 or 15 or 16 or 17

19. 10 and 18

\section{CONTRIBUTIONSOFAUTHORS}

CC drafted the protocol. All authors participated in reviewing the manuscript. All authors read and approved the final manuscript.

\section{DECLARATIONSOF INTEREST}

CC, CS and NA are investigators on studies likely to be included in the review. These authors will not assess their own studies for inclusion or risk of bias and will not extract data from their own studies. 